\title{
Reinventing a primordial hydrogenase with a di-Nickel center
}

JENNIFER TIMM, JOSHUA MANCINI, DOUGLAS PIKE, ALEXEI TYRYSHKIN, SAROJ POUDEL, VIKAS NANDA AND PAUL FALKOWSKI

Rutgers University

Presenting Author: jennifer.timm@marine.rutgers.edu

Today, metabolism and catalysis in every organism is carried out by a complex set of proteins. However, at the beginning when life emerged and abiotic processes transition to biotic ones, the proteins and molecules involved must have been a lot simpler than what we find today. While presumably simpler than today, the fundamental chemical and physical processes are most likely highly similar if not identical. One of the most ancient processes for energy production and transfer in living organisms is the reversible oxidation of molecular hydrogen, a molecule highly abundant in early earth's atmosphere. The protein catalyzing this reaction is hydrogenase. Simple, primordial versions of hydrogenase are likely to have occurred very early on in the emergence of life and our work is aimed to reconstruct a simple peptide molecule that could have been at the very beginning of hydrogenase evolution. We designed a peptide based on the chemical requirements necessary to emulate a hydrogenase active site, binding Nickel ions. We report the successful design of a nickel-binding peptide capable of robustly producing molecular hydrogen from protons under a wide variety of conditions. Biophysical and structural investigation strongly indicate the peptide to form a di-nickel cluster analogous to Acetyl-CoA synthase, an ancient protein central to metabolism in all life forms. This could indicate the ability to catalyze multiple different reactions and supports the hypothesis that early life forms might have started off using simpler peptide-based molecule generalists. 